EESTI NSV TEADUSTE AKADEEMIA TOIMETISED. XI KÖIDE FOOSIKALIS-MATEMAATILISTE JA TEHNILISTE TEADUSTE SEERIA. 1962, NR. 1

ИЗВЕСТИЯ АКАДЕМИИ НАУК ЭСТОНСКОИ ССР. ТОМ ХI СЕРИЯ ФИЗИКО-МАТЕМАТИЧЕСКИХ И ТЕХНИЧЕСКИХ НАУК. 1962, № 1

\title{
О МНОЖИТЕЛЯХ СУММИРУЕМОСТИ ДЛЯ МЕТОДА ЧЕЗАРО ОТРИЦАТЕЛЬНОГО ПОРЯДКА
}

\author{
C. БАРОН, \\ кандидат физико-математических наук
}

Т. ТАММАИ

В настоящей заметке* изучается вопрос о множителях суммируемости как простых, так и двойных рядов, когда ряды $\sum \varepsilon_{n} u_{n}$ и $\sum_{m, n} \varepsilon_{m n} u_{m n}$ предполагаются суммируемыми или абсолютно суммируемыми методом Чезаро отрицательного порядка. Ввиду того, что метод Чезаро отрицательного порядка (для простых рядов и ограниченных двойных рядов) слабее даже метода сходимости, условия приводимых ниже теорем проще соответствующих теорем для положительного порядка суммирования.

\section{§ 1. Простые ряды}

Те орем а 1. Если $\alpha \geqslant 0,-1<\beta<0$, то для того, чтобы числа $\varepsilon_{n}$ были множителями суммируемости типа $\left(\left|C^{2}\right|, C^{\beta}\right)$ или $\left(\left|C^{\alpha}\right|,\left|C^{\beta}\right|\right)$, необходимо и достаточно условие

$$
\varepsilon_{n}=O\left[(n+1)^{\beta-\alpha}\right] .
$$

Т еорема 2. Если $\alpha \geqslant 0,-1<\beta<0$, то для того, чтобы числа $\varepsilon_{n}$ были мнояителями суммиругмости типа $\left(C^{\alpha},\left|C^{\beta}\right|\right)$ или $\left(C_{O}^{\alpha},\left|C^{\beta}\right|\right)$, необходимо и достаточно условие

$$
\sum(n+1)^{\alpha-\beta}\left|\varepsilon_{n}\right|<\infty
$$

Доказательство теоремы 1. Необходиость условия теоремы вытекает непосредственно из условия (2) статьи [1]. Доказательство его достаточности покрывается случаем 1 доказательства теоремы 1 статьн [1] (стр. 51), ибо в нашем случае $i+\beta-a-1<-1$ для $i=0, \ldots, a$.

Доказательство теоремы 2. Необходимость условия теоремы легко вывесุти непосредственно из условия (24) статьи [']. Доказательство его достаточности исчерпывается случаем 1 доказательства теоремы 4 статьи ['] (стр. 62), ибо в данном случае $i+\beta-a-2<-1$ при $i=0, \ldots, a+1$.

* Поскольку настоящая заметка тесно примыкает к статьям [1-3], в ней сохраняются определения и обозначения, использованные в них, а также, в целях краткссти нзложения, приводятся ссылки на доказательства, имеющиеся в названных статьях. 


\section{§2. Двойные ряды}

Т еорем а 3. Если $\alpha, \beta \geqslant 0$, то для того, чтобы числа $\varepsilon_{m_{n}}$ были множителями суммируемости типов а) $\left(C_{l}^{\alpha, \beta}, C^{\gamma, \hat{\gamma}}\right)$,

г) $\left(C_{l}^{\alpha, \beta}, C_{l}^{\gamma, \hat{\delta}}\right)$, необходимы и достаточны

б) $\left(C_{l}^{\alpha, \beta}, C_{b}^{7,3}\right)$,

в) $\left(C_{l}^{\alpha, \beta}, C_{r}^{\gamma, \delta}\right)$,

1) в случае $-1<\gamma, \delta<0$ условие

$$
\varepsilon_{m n}=O\left[(m+1)^{\gamma-\alpha}(n+1)^{\hat{\delta}-\beta}\right] ;
$$

2) в случае $\gamma \geqslant 0,-1<\delta<0$ условие

$$
\Delta_{m}^{\alpha} \varepsilon_{m n}=O\left[(m+1)^{-\alpha}(n+1)^{\hat{i}-\beta}\right]
$$

и при $\gamma<\alpha$ условие (D), при $\gamma \geqslant \alpha$ условие

$$
\varepsilon_{m n}=O\left[(n+1)^{\delta-\beta}\right]
$$

3) в случае $-1<\gamma<0$, $8 \geqslant 0$ условие

$$
\Delta_{n}^{\beta} \varepsilon_{m n}=O\left[(m+1)^{\gamma-\alpha}(n+1)^{-\beta}\right]
$$

и при $\delta<\beta$ условие (D), при $\delta \geqslant \beta$ условие

$$
\varepsilon_{m n}=O\left[(m+1)^{\gamma-\alpha}\right] .
$$

Те орем а 4. Если $\alpha, \beta \geqslant 0$, то для того, итобы числа $\varepsilon_{m n}$ были множителяяи суммируемости типов а) $\left(C_{O}^{\alpha, \beta}, C_{l}^{\gamma, \hat{\jmath}}\right)$, б) $\left(C_{b}^{\alpha, \beta}, C_{l}^{\gamma, \delta}\right)$, в) $\left(C_{r}^{\alpha, \beta}, C_{l}^{\gamma, \hat{\jmath}}\right)$, необходимь и достаточны

1) в случае $-1<\gamma, \delta<0$ условие

$$
\sum_{m, n}(m+1)^{\alpha-\gamma}(n+1)^{\beta-\sigma}\left|\varepsilon_{m n}\right|<\infty ;
$$

2) в случае $\gamma \geqslant 0,-1<\delta<0$ условие

$$
\sum_{m, n}(m+1)^{\alpha}(n+1)^{\beta-\beta}\left|\Delta_{m}^{\dot{\alpha}+1} \varepsilon_{m n}\right|<\infty
$$

и при $\gamma<\alpha+1$ условие (М), при $\gamma \geqslant \alpha+1$ условие

$$
\sum_{m, n}(m+1)^{-1}(n+1)^{\beta-\grave{\iota}}\left|\varepsilon_{m n}\right|<\infty ;
$$

3) в случае $-1<\gamma<0$, $8 \geqslant 0$ yсловие

$$
\sum_{m, n}(m+1)^{\alpha-\gamma}(n+1)^{\beta}\left|\Delta_{n}^{\beta+1} \varepsilon_{m n}\right|<\infty
$$

и при $\delta<\beta+1$ условие (М), при $о \geqslant \beta+1$ условие

$$
\sum_{m, n}(m+1)^{\alpha-\gamma}(n+1)^{-1}\left|\varepsilon_{m n}\right|<\infty .
$$

Доказательсто теоремы 3. Необходимость условий теоремы вытекает нз доказательства леммы 4-статьи [2]. Достаточность в случае 1) доказывается точно так же, как оценка первого слагаемого схемы (19) статьи $\left[{ }^{2}\right]$, а, например, в случае 2) - как оценки первого, второго и пятого слагаемых схемы (19) статьи [2], ибо в данном случае эта схема заменяется следующей: 


$$
\sum_{i, j=0}^{a, b} B_{2}^{j} B_{1}^{i}=\left(\sum_{i, j=0}^{P, b}+\sum_{i, j=a=c+1,0}^{a, b}\right) B_{2}^{i} B_{1}^{i}+\sum_{j=0}^{b} B_{2}^{j} B_{1}^{a-c}
$$

Доказательств о теоремы 4. Необходимость условий теоремы следует из доказательства леммы 2 статьи [3]. Достаточность в случае 1) доказывается точно так же, каќ оценка первого слагаемого схемы (20) статьн [3], а, например, в случае 2) - как оценки первого, второго и пятого слагаемых схемы (20) статьи [3], нбо в последнем случае эта схема заменяется следующей:

$$
\sum_{i, j=0}^{a+1, b+1} B_{2}^{\prime j} B_{1}^{\prime i}=\left(\sum_{i, j=0}^{P+1, b+1}+\sum_{i, j=a=c+2,0}^{a+1, b+1}\right) B_{2}^{\prime j} B_{1}^{i}+\sum_{j=0}^{b+1} B_{2}^{\prime j} B_{1}^{\prime a-c+1}
$$

3 а ме чан пе. Положив в часть 1) теоремы $3 \quad a=\beta=0$ и $\varepsilon_{m n}=\left(A_{m}^{\sigma} A_{n}^{\tau}\right)^{-1}$ получаем следующую теорему Жака-Тимана ([$\left.{ }^{4}\right]$, стр. 37):

Если ряд $\sum_{m, n} u_{m n}$ абсолютно сходится, то ряд $\sum_{m, n}\left(A_{m}^{\sigma} A_{n}^{\bar{z}}\right)^{-1} u_{m n}$ суммируем методом $C_{l}^{-\sigma,-\tau} ; 0<\sigma, \tau<1$.

\section{Л ИТЕРА Т У РА}

1. С. Б арон, Новые доказательства основных теорем о множителях суммируемости, Изв. АН Эст. ССР, Сер. физ.-мат. и техн. наук, т. IX, № 1, 1960, $47-68$.

2. С. Барон, Множители суммируемости и абсолютной суммируемости для двойных рядов, абсолютно суммируемых методом Чезаро, Уч. зап. Тартуск. ун-та, 102, 1961, $118-134$.

3. С. Барон, Множители абсолютной суммируемости для Чезаро-суммируемых н Чезаро-ограниченных двойных рядов, Уч. зап. Тартуск. ун-та, 102, 1961 $135-155$.

4. И. Е. Ж ак, М. Ф. Т и м н, О суммированни двойных рядов, Матем. сб., 35 (77), 1954, 21-56.

Тартуский государственный университет

Поступила в редакцию

5. X 1960

\section{SUMMEERUVUSTEGURITEST NEGATIIVSET JARKU CESARO MENETLUSE JAOKS}

\section{S. Baron,}

füüsikalis-matemaatiliste teaduste kandidaat

\section{T. Tammai}

\section{Resümee}

Vaadeldakse nii harilike kui ka kahekordsete ridade korral mitmesuguseid summeeruvustegurite tüüpe Cesàro menetluse jaoks juhul, kus nõutakse ridade $\Sigma \varepsilon_{n} u_{n}$ ja $\Sigma \varepsilon_{m n} u_{m n}$ negatiivset järku summeeruvust vôi absoluutset summeeruvust; ridade $\Sigma u_{n}$ ja $\Sigma u_{m n}$ kohta käsitletakse mittenegatiivset järku Cesàro menetlusega tōkestatust, summeeruvust või absoluutset summeeruvust. Saadud teoreemide tōestused põhinevad vastavatel tõestustel eelmistest artiklitest. Teoreemist 3 tuleneb erijuhuna Zak-Timani tuntud lause [ $\left.{ }^{4}\right]$. 


\section{OBER SUMMIERBARKEITSFAKTOREN BEI CESAROVERFAHREN NEGATIVER ORDNUNG}

\section{S. Baron}

\section{T. Tammai}

\section{Zusammenfassung}

Es werden einige Typen von Summierbarkeitsfaktoren für gewöhnliche Reihen und für Doppelreihen behandelt. Untersucht wird der Fall, dass die Reihen $\Sigma \varepsilon_{n} u_{n}$ und $\Sigma \varepsilon_{m n} u_{m n}$ negativer Ordnung Cesàro-summierbar oder absolut summierbar sind, die Reihen $\Sigma u_{n}$ und $\Sigma u_{m_{n}}$ jedoch nichtnegativer Ordnung Cesàro-beschränkt, Cesàro-summierbar oder absolut summierbar sind. Die Beweise der Sätze beruhen auf entsprechenden Beweisen aus den Arbeiten [1-3]. Aus Theorem 3 folgert sich als Sonderfall ein bekannter Satz von ZakTiman $\left[{ }^{4}\right]$.

Staatsuniversität zu Tartu

Eingegangen

am 5. Okt. 1960 